\title{
SOPORTE CIRCULATORIO MECÁNICO CON BOMBA CENTRÍFUGA
}

\author{
MECHANICAL CIRCULATORY SUPPORT WITH CENTRIFUGAL PUMP
}

\author{
ALEJANDRO M. BERTOLOTTI'
}

\section{RESUMEN}

La insuficiencia cardíaca avanzada es una entidad compleja con prevalencia en aumento. Su abordaje incluye la combinación de estrategias farmacológicas, quirúrgicas y, cuando se agotan las opciones terapéuticas, terapias de destino o reemplazo, siendo estas últimas principalmente los dispositivos de asistencia circulatoria mecánica y el trasplante cardíaco. Las bombas centrífugas se destacan en nuestro medio como una alternativa de asistencia a corto/mediano plazo como puente a la decisión, a la recuperación o al trasplante cardíaco, permitiendo un soporte uni- o biventricular a estos pacientes críticos.

Palabras clave: corazón auxiliar, shock cardiogénico, cuidados críticos

\section{ABSTRACT}

Advanced heart failure is a complex entity with an increasing prevalence. Its approach includes the combination of pharmacological, surgical strategies and, when the therapeutic options are exhausted, target or replacement therapies, the latter being mainly mechanical circulatory assistance devices and cardiac transplantation. Centrifugal pumps stand out in our area as an alternative of short/medium term assistance as a bridge to decision, recovery or heart transplantation, allowing uni or biventricular support to these critical patients.

Keywords: heart-assist devices, cardiogenic shock, critical care

REVISTA CONAREC 2018;33(147):279-282 | DOI:10.32407/RCON/2018147/0279-0282

\section{INTRODUCCIÓN}

La insuficiencia cardíaca avanzada es una entidad compleja con prevalencia en aumento. Su abordaje incluye la combinación de estrategias farmacológicas, quirúrgicas y, cuando se agotan las opciones terapéuticas, terapias de destino o reemplazo, siendo estas últimas principalmente los dispositivos de asistencia circulatoria mecánica (ACM) y el trasplante cardíaco?

Los dispositivos de ACM pueden clasificarse de distintas maneras tomando en cuenta diversas características como la durabilidad, el flujo y su ubicación anatómica, entre otros². En los pacientes con shock cardiogénico refractario al tratamiento médico, se prefieren los dispositivos de corto-mediano plazo para la rápida estabilización del paciente, como puente a una recuperación, a un dispositivo de largo plazo como terapia de destino o al trasplante cardíaco. En este grupo, se destacan en nuestro medio las bombas centrífugas (BC) de flujo continuo.

\section{DEFINICIÓN Y CARACTERÍSTICAS}

Las bombas centrífugas son dispositivos de uso temporario de corto plazo, para asistencia circulatoria mecánica en pacientes con un cuadro de shock cardiogénico severo refractario a otras terapias. Su im-

1. Jefe del Servicio de Trasplante Intratorácico e Insuficiencia Cardíaca. Hospital Universitario Fundación Favaloro, CABA.

$\triangle$ Correspondencia: Dr. Alejandro Mario Bertolotti. Av. Belgrano 1746 C1093AAS CABA, Rep.Argentina. bertolotti.alejandro66@gmail.com

Los autores declaran no poseer conflictos de intereses.

Recibido: 01/11/2018|Aceptado: 10/11/2018 plante requiere un procedimiento quirúrgico y permite asistir independientemente las funciones ventriculares izquierda, derecha o ambas (soporte uni- o biventricular). La interposición de una membrana oxigenadora extracorpórea permite la conversión a sistema de soporte vital con oxigenación sanguínea (ECMO).

Las BC proveen flujo de tipo radial y continuo, no pulsátil, mediante la generación de un diferencial de presión entre el sitio de ingreso y egreso de la sangre a la carcasa de la bomba, gracias a la rotación a altas velocidades del propulsor central.

Estos dispositivos requieren del uso de anticoagulación y/o antiagregación permanente, ya que el contacto de la sangre con elementos no biológicos (cánulas, tubuladuras, carcasa y propulsor) y las turbulencias que se generan en la circulación de la misma favorecen los fenómenos trombóticos por activación de la cascada de coagulación.

\section{SISTEMAS DISPONIBLES}

El sistema de soporte circulatorio agudo CentriMag ${ }^{\mathrm{TM}}$, se caracteriza por incorporar la tecnología de flujo Full-MagLev ${ }^{T M}$ mediante levitación magnética del propulsor permitiendo una mejor performance hemodinámica al evitar puntos de contacto entre la carcasa y el propulsor (Figura 1). Esta característica de funcionamiento disminuye notablemente las fuerzas de corte y fricción que favorecen la hemólisis y evita zonas de mayor turbulencia y estasis sanguíneas que generan mayor probabilidad de formación de trombos ${ }^{3,4}$. Este dispositivo cuenta con dos modelos de cabezal centrífugo, uno para niños hasta 10 kg de peso (PediVAS CentriMag ${ }^{\text {TM}}$ ) y otro para pacientes con mayor superficie corporal, que permite generar flujos de hasta 9,9 litros/min. Su utilización ha sido aprobada por un período de hasta 30 días, aunque existen reportes de su utilización por más de 90 días sin necesidad de cambios del dispositivo por complicaciones mayores o fallas de funcionamiento ${ }^{5}$. Esta tecnología ha sido utilizada en Argentina 

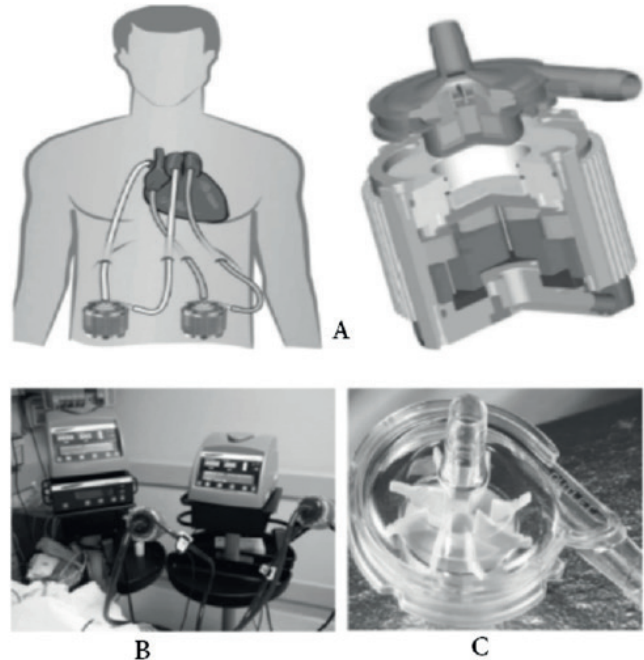

Figura 1. A. Dispositivo de asistencia biventricular y bomba con rotor. B. Consola con dos rotores electromagnéticos para asistencia biventricular. C. Bomba centrífuga. Modificado de Bertolotti AM et al. Experiencia con bomba centrífuga magnética en pacientes con shock cardiogénico (INTERMACS 1). Rev Argen Cardiol 2014;82(3):205-10.

desde el año $2007^{6}$ y cuenta para su uso con aprobación mediante resolución de ANMAT?

Existen otros dispositivos de BC para soporte circulatorio mecánico agudo disponibles en el país, como la bomba RotaFlow ${ }^{\mathrm{TM}}$ (Maquet, Getinge Group, US) y el sistema Revolution ${ }^{\text {TM }}$ (LivaNova PLC, UK). Estas BC, con características similares de funcionamiento y soporte hemodinámico, no utilizan la tecnología de levitación magnética.

\section{TÉCNICA QUIRÚRGICA DE IMPLANTE}

El implante de las BC para soporte ventricular derecho, izquierdo o biventricular requiere de un procedimiento quirúrgico cardiovascular central, mediante esternotomía mediana y utilización de circulación extracorpórea (CEC) para permitir las maniobras necesarias de canulación sin generar mayor compromiso hemodinámico en pacientes con shock cardiogénico.

Los sitios de colocación de las cánulas de egreso e ingreso varían según el tipo de soporte a implementar.

Para asistencia ventricular izquierda (AVI), la cánula de drenaje (o de ingreso al dispositivo) puede ser colocada directamente en la aurícula izquierda o en el ápex del ventrículo izquierdo (VI). La elección del sitio de canulación dependerá de la estrategia definida (p. ej.: puente a la recuperación con posible explante posterior, puente a la decisión o puente al trasplante), de la patología de base y causa del shock cardiogénico (p. ej.: miocardiopatía con ventrículo izquierdo restrictivo que limite el acceso a la cavidad ventricular, miocarditis aguda potencialmente recuperable, infarto agudo de miocardio) y/o antecedentes del paciente (p. ej.: cirugía cardíaca previa). Se han descrito nuevas técnicas para un implante seguro y hemostático en el ápex. La cánula de infusión (o egreso del dispositivo) se implanta en la aorta ascendente directamente o mediante interposición de una prótesis vascular. Se han descrito estrategias de AVI mediante técnicas quirúrgicas miniinvasivas que evitan el uso de CEC, accediendo al ápex del VI por una toracotomía mínima anterior izquierda. Para acceso de la aorta se realiza una esternotomía mínima parcial o canulación en alguna rama arterial central como las arterias axilares/subclavias derecha o izquierda.

Para asistencia ventricular derecha (AVD), la cánula de drenaje se implanta en la aurícula derecha (AD) por acceso directo a través de la pared libre de la misma. Otra opción es llegar a la AD por vía percutánea a través de los sistemas de venas cava superior o inferior, mediante punción de venas femorales o yugulares internas. La cánula de infusión, se implanta en el tronco de la arteria pulmonar.

Para asistencia biventricular (ABiV), se emplea la combinación de ambas técnicas de AVI y AVD.

\section{OBJETIVOS DE LA ACM Y MANEJO HEMODINÁMICO}

Los objetivos de la ACM con BC son:

- Lograr una adecuada perfusión y oxigenación de todos los sistemas orgánicos ajustado a la superficie corporal $(60 \mathrm{ml} / \mathrm{kg}$ de peso en adultos) y al cuadro actual del paciente.

- Lograr una adecuada descarga de las cavidades cardíacas afectadas (VI, ventrículo derecho [VD] o ambos) y disminución del estrés parietal del miocardio.

Todos los pacientes requieren monitorización invasiva con catéteres arteriales y venosos centrales de Swan Ganz para obtener información respecto de la precarga y la poscarga del VD e indirectamente la presión de precarga del VI mediante la presión de enclavamiento pulmonar. La presión venosa central idealmente debe mantenerse en un rango entre 10 y $15 \mathrm{mmHg}$ tanto para AVI, AVD o ABiV, de manera de asegurar una precarga adecuada. Las presión arterial media debe estar idealmente entre 60 y $70 \mathrm{mmHg}$ con precarga adecuada y resistencias vasculares entre 800 y $1200 \mathrm{dyn} \cdot \mathrm{s} \cdot \mathrm{cm}^{-5}$ La monitorización de la presión de la arteria pulmonar permite establecer estrategias de vasodilatación pulmonar (uso de óxido nítrico inhalatorio; o drogas inodilatadoras como milrinona o dobutamina) a los fines de disminuir la poscarga del VD y asegurar el correcto llenado de la Al. Esta estrategia favorece tanto el soporte de la función del VD en caso de AVI, como el correcto funcionamiento de la AVD o ABiV.

La utilización de ecografía cardíaca transesofágica (ETE) intraoperatoria es mandatoria para la correcta evaluación de la hemodinamia del paciente bajo soporte con ACM. Es necesario valorar el correcto posicionamiento de las cánulas de drenaje tanto en cavidades auriculares como en VI, ya que podrían originarse obstrucciones mecánicas del drenaje por inadecuado posicionamiento que deben ser corregidas en el quirófano (p. ej.: succión de las valvas de las válvulas auriculoventriculares, succión y obstrucción del extremo de las cánulas por cercanía de las paredes de las cámaras drenadas). El ETE permite además la valoración de la precarga (llenado y/o vaciado) de las cámaras cardíacas asistidas. La rectificación y posicionamiento central del septum interventricular es un indicador de adecuado drenaje de las cámaras, además de favorecer la función sistólica del VD en caso de AVI. Una cánula implantada incorrectamente o un llenado inadecuado de las cámaras drenadas pueden oca- 
TABLA 1. Indicaciones de ACM.

$\begin{array}{ll} & \text { Uso a corto plazo de la ACM (como SVE o ECMO) } \\ & \text { en pacientes con shock cardiogénico hasta } \\ & \text { que se estabilice el estado hemodinámico y la } \\ \text { Puente a la decisión/ } & \text { perfusión de órganos finales; se excluyen las } \\ \text { puente al puente } & \text { contraindicaciones de la ACM a largo plazo (daño } \\ & \text { cerebral tras reanimación) y se pueden evaluar } \\ & \text { otras opciones terapéutica, como el DAV o el } \\ & \text { trasplante cardíaco. }\end{array}$

sionar problemas de funcionamiento, succión y colapso de la cámara, aumento de las presiones negativas generado por el dispositivo y formación de burbujas por el fenómeno de cavitación; con consecuencias graves de embolia aérea. La evaluación de la función ventricular por ETE permite establecer estrategias de asistencia univentricular o biventricular. Los parámetros más utilizados para valorar la función del VD son el desplazamiento sistólico del plano del aniIlo tricuspídeo (siglas en inglés, TAPSE), la integral velocidad tiempo (siglas en inglés, VTI) y el grado de suficiencia de la válvula tricuspídea. La confirmación de suficiencia valvular aórtica e indemnidad de los septum auricular y ventricular es necesaria para asegurar un correcto soporte circulatorio mecánico, evitando la recirculación y/o posibles embolias paradójicas.

\section{INDICACIONES DE ACM}

Las guías de la Sociedad Europea de Cardiología para el diagnóstico y tratamiento de la insuficiencia cardíaca aguda y crónica publicadas en 2016, describen las indicaciones actuales para el uso de ACM en pacientes con diagnóstico de falla cardíaca (Tabla 1$)^{9}$.

Las guías para soporte circulatorio mecánico de la Sociedad Internacional de Trasplante Cardíaco y Pulmonar establecen que todos los pacientes candidatos a ACM deben tener determinada su CF (NYHA) y ser clasificados según los perfiles hemodinámicos del registro INTERMACS (Recomendación Clase I, Nivel de evidencia: C) ${ }^{10}$. Si bien estas recomendaciones fueron establecidas fundamentalmente para dispositivos implantables de largo plazo, son apli- cables al manejo integral de pacientes candidatos a ACM con dispositivos de corto plazo. En Argentina el acceso a este tipo de tecnología se encuentra muy limitado, fundamentalmente debido al alto costo que conlleva su aplicación en un complejo sistema de salud basado en multipagadores. Situación similar se presenta en otros países de la región Latinoamericana, por lo que los dispositivos de menor costo como las BC se han transformado en una alternativa válida. Se han reportado experiencias favorables de ACM con CentriMag ${ }^{\text {TM }}$ en Argentina ${ }^{6,11,12}$, Chile ${ }^{13,14}$, Colombia ${ }^{15}$ y Brasi $^{16}{ }^{16}$ como puente a la recuperación, puente a la decisión y puente al trasplante/retrasplante cardíaco.

\section{COMPLICACIONES DE LA ACM CON BC}

Las complicaciones y efectos adversos en pacientes bajo soporte circulatorio son comunes a todos los dispositivos de ACM, ya sean temporarios o permanentes, y están relacionados con:

- El procedimiento de implante y canulación, ya sea central o accesos vasculares periféricos (taponamiento, sangrado aumentado posoperatorio, isquemia distal a la canulación en miembros).

- La necesidad de anticoagulación y/o antiagregación plaquetaria que favorece los procesos hemorrágicos y/o tromboembólicos, fundamentalmente los eventos neurológicos.

- Las infecciones asociadas a la interfase de la piel con la exteriorización de cánulas.

- Fallas mecánicas y la hemólisis provocada por los dispositivos.

En un metaanálisis de la tasa de eventos adversos en pacientes bajo soporte con BC CentriMag ${ }^{\text {TM }}$ publicado por Bosizenko et al., la media de ocurrencia de sangrado fue del 28\% (IC95\%: 23-32), de trombosis 7\% (IC95\%: 5-11\%), hemólisis 3\% (IC95\%: 1-6\%), complicaciones neurológicas 7\% (IC95\%: 4-11\%), infecciones 24\% (IC95\%: 19 30\%), complicaciones renales 28\% (IC95\%: 22-36\%) y falla del dispositivo 0,08\% (IC95\%: 0,0-0,5\%; 3 casos sobre 512 pacientes). La comparación de estos eventos entre las poblaciones adulta y pediátrica demostró que la tasa de eventos hemorrágicos (23 vs. 46\%) y trombosis (4 vs. 22\%) fue significativamente mayor en los pacientes pediátricos ${ }^{17}$.

\section{CONCLUSIÓN}

Actualmente, la insuficiencia cardíaca avanzada es una entidad compleja que requiere de un enfoque integral, siendo a veces necesario el sostén hemodinámico mediante dispositivos de asistencia circulatoria mecánica de corto o mediano plazo como puente a la decisión, a la recuperación o al trasplante cardíaco. Las BC constituyen una alternativa válida en nuestro medio que permiten un soporte uni o biventricular a estos pacientes críticos. 


\section{BIBLIOGRAFÍA}

1. Kozik DJ, Plunkett MD. Mechanical circulatory support. Organogenesis 2011;7(1):50-63.

2. Nagpal AD, Singal RK, Arora RC, Lamarche Y. Temporary Mechanical Circulatory Support in Cardiac Critical Care: A State of the Art Review and Algorithm for Device Selection. Can J Cardiol 2017;33(1):110-8

3. De Robertis F, Briks EJ, Rogers P, Dreyfus G, Pepper JR, Khaghani A. Clinical performance with the Levitonix CentriMag short-term ventricular assist device. J Heart Lung Transplant 2006;25(2):181-6.

4. Zhang J, Gellman B, Koert KA, Dasse KA, Gilbert RJ, Griffith BP, et al. Computational and experimental evaluation of the fluid dynamics and hemocompatibility of the CentriMag blood pump. Artif Organs 2006;30(3):168-77.

5. Mohite PN, Zych B, Popov AF, Sabashnikov A, Sáez DG, Patil NP, et al. CentriMag short term ventricular assist as a bridge to solution in patients with advanced heart failure: use beyond 30 days. Eur J Cardiothorac Surg 2013;44(5):e310-5.

6. Favaloro RR, Bertolotti A, Diez M, Favaloro L, Gómez C, Peradejordi M, et al. Adequate systemic perfusion maintained by a CentriMag during acute heart failure. Tex Heart Inst J 2008;35(3):334-9.

7. Disposición 1509-14 ANMAT wWw.anmat.gov.ar/boletin_anmat/marzo_2014/ Dispo_1509-14.pdf

8. Shen TC, Tsai KT, Hu CY, Chen RJ. Skirted Cannula Technique for Apical Cannulation in Implantation of Centrimag Left Ventricular Assist Device. Ann Thorac Surg 2016;101(6):2404-6.

9. Ponikowski P, Voors AA, Anker SD, Bueno H, Cleland JG, Coats AJ, et al. 2016 ESC Guidelines for the diagnosis and treatment of acute and chronic heart failure: The Task Force for the diagnosis and treatment of acute and chronic heart failure of the European Society of Cardiology (ESC). Eur Heart J 2016;37(27):2129-200.
10. Feldman D, Pamboukian SV, Teuteberg JJ, Birks E, Lietz K, Moore SA. The 2013 International Society for Heart and Lung Transplantation Guidelines for mechanical circulatory support: Executive summary. J Heart Lung Transplant 2013;32(2):157-87.

11. Bertolotti AM, Peradejordi M, Favaloro L, Carballo M, Absi D, Favaloro LE, et al. EXperiencia con bomba centrífuga magnética en pacientes con shock cardiogénico (INTERMACS 1). Rev Argent Cardiol 2014;82(3):205-10.

12. Cohen Arazi $H$, Spampinato $R$, Waldman SV Rodríguez W, Grancelli H, Schnetzer $\mathrm{N}$, et al. Asistencia circulatoria temporaria como puente al retrasplante. Rev Argent Cardiol 2010;78(6):516-7.

13. Villavicencio M, Larrea R, Larraín E, Turner F, Rivera J, Peralta JP, et al. Puente al trasplante de 4 semanas utilizando el sistema de asistencia ventricular Levitronix Centrimag ${ }^{\oplus}$ en el shock cardiogénico post-infarto al miocardio. Caso clínico. Rev Med Chile 2010:138(6):752-757.

14. Villavicencio M, Larraín E, Larrea R, Peralta JP, Lim JS, Rojo P, et al. Bridge to trans plant or recovery in cardiogenic shock in a developing country. Asian Cardiovasc Thorac Ann 2017;25(2):105-12.

15. Garzon-Rodriguez JD, Obando-Lopez C, Giraldo-Grueso M, Sandoval-Reyes N, Camacho J, Umaña JP. Mechanical circulatory support as bridge therapy for heart transplant: case series report. BMC Research Notes 2018;11(1):430.

16. Breda JR, Gaia DF, Macedo M, Motta P, Martins M, Alves D, et al. Ventricular assist device implantation with CentriMag VAS ${ }^{\circledR}$ for biventricular mechanical support. Rev Bras Cir Cardiovasc 2013;28(3):401-4.

17. Borisenko O, Wylie G, Payne J, Bjessmo S, Smith J, Yonan N, et al. Thoratec CentriMag for Temporary Treatment of Refractory Cardiogenic Shock or Severe Cardiopulmonary Insufficiency: A Systematic Literature Review and Meta-Analysis of Observational Studies. Asaio Journal 2014;60(5):487-97. 